EDITORIAL

\title{
Immunometabolism at the intersection of metabolic signaling, cell fate, and systems immunology
}

(c) The Author(s), under exclusive licence to CSI and USTC 2022

Cellular \& Molecular Immunology (2022) 19:299-302;

https://doi.org/10.1038/s41423-022-00840-x

Metabolism is a core process underlying essentially all biological functions. The integration of metabolism with immunity, known as immunometabolism, is at the forefront of immunology research, as it continues to transform the field. Research over the past decade has established metabolism as a fundamental requirement underlying immune system function in health and disease. The critical link between metabolism and immunity is highlighted by the findings that cellular metabolism affects immune cell state and fate, and contributes to infectious disease, inflammation, and cancer [1]. Further, interactions between the immune and metabolic systems mediate organismal homeostasis [2].

Several principles have recently emerged in immunometabolism. Extensive interplay exists between metabolism-associated environmental cues (e.g., nutrients) and immune receptors, which act in synergy to mediate innate and adaptive immune responses. The outcome of immune reactions is further shaped by the crosstalk between signal transduction and metabolic reprogramming in a process known as bidirectional metabolic signaling [3]. Such metabolic signaling serves as a driver of immune cell state and fate and tissue immunity, evidenced by recent findings in
T cells, B cells, dendritic cells (DCs), and macrophages, among other cells. Finally, as metabolism functions as highly complex networks for both cell signaling and cell-cell and cell-environment interactions, there is increasing appreciation of intracellular and intercellular networks for immunometabolism, the latter of which is exemplified by the extensive crosstalk between the immune system, adipose tissue, and diet [4]. Collectively, understanding immunometabolism at the signaling, cellular, and systems levels has advanced our understanding of immunity and metabolism and provided new opportunities for therapeutic targeting of immune-mediated disease, cancer, and metabolic disorder (Fig. 1). This special issue of Cellular \& Molecular Immunology on immunometabolism compiles a total of ten review articles from leading experts in the field that summarize these emerging principles and discuss how an integrative understanding of immunometabolism promises to reveal new biology and disease therapies.

\section{PATHWAYS: BIDIRECTIONAL METABOLIC SIGNALING IN INNATE AND ADAPTIVE IMMUNITY}

Cells in the innate and adaptive immune systems are characterized by rapid transition between the quiescent and activated states, marked heterogeneity of cell fate choices, and context-specific

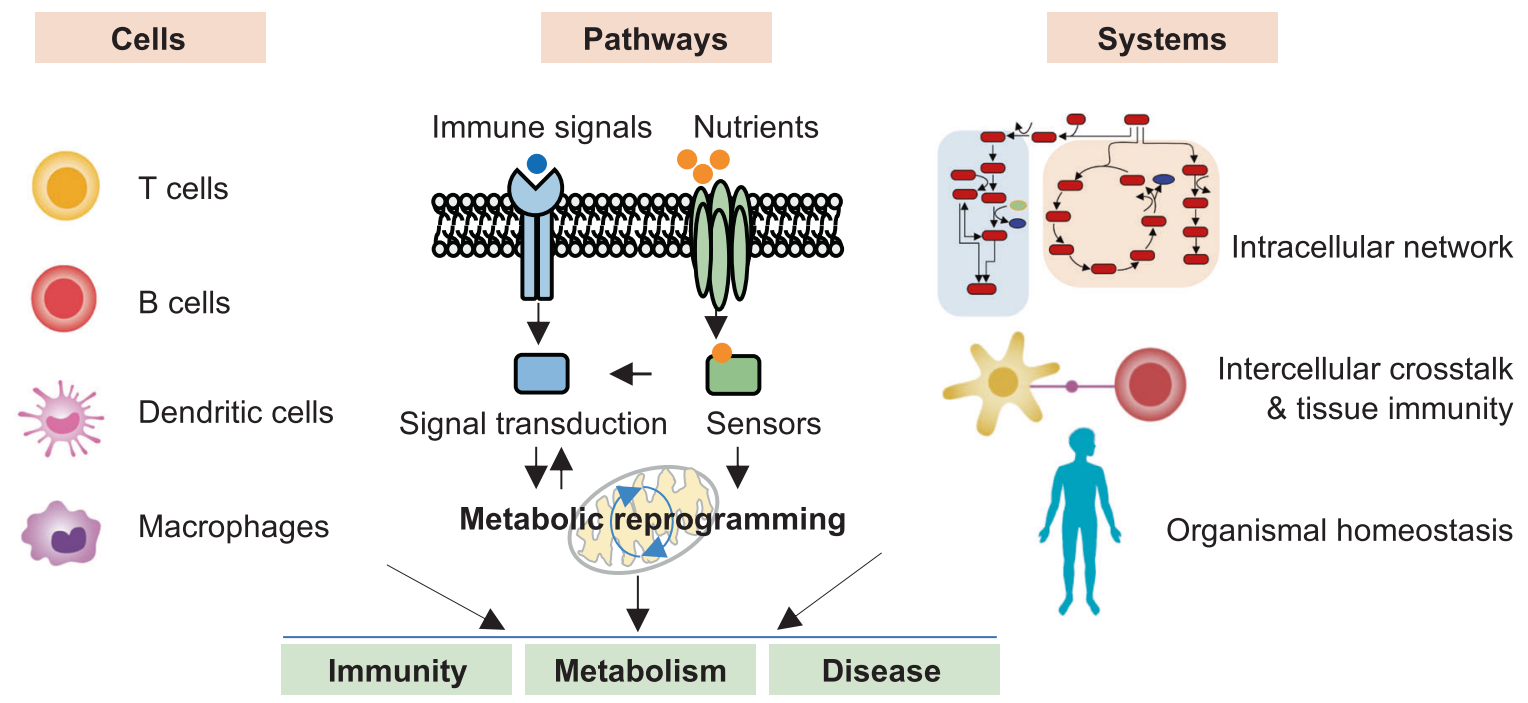

Fig. 1 An integrative view of immunometabolism to link metabolic pathways, immune cell biology, and systemic metabolism. Center: metabolic pathways in T cells are mainly discussed by the reviews from Dr. Cantrell, Dr. Rathmell, and colleagues, while those in innate immunity are from Dr. Bensinger, Dr. Ting, and colleagues. Left: immunometabolism of B cell biology, dendritic cell biology, and macrophage biology are mainly discussed by the reviews from Drs. Boothby, Ho, and Sancho, respectively. Right: metabolic network is discussed by the review from Dr. Kuchroo and colleagues, and cell-cell crosstalk and organismal metabolism are discussed by the reviews from Dr. Kallies, Dr. Lynch, and colleagues. 
tissue adaptation. Associated with such temporospatial regulation of immune reactions is the dynamic reprogramming of cell metabolism and the crosstalk with signal transduction, including the signaling roles mediated by metabolites and nutrients.

Focusing on T cells, a central cell type in adaptive immunity, the reviews from Dr. Doreen Cantrell [5], Dr. Jeffrey Rathmell [6], and colleagues discuss the intrinsic and extrinsic mechanisms for metabolic controls, respectively. T cell activation is accompanied by a pronounced increase in cell size and induction of activation markers, followed by cell cycle entry, clonal expansion, and lineage differentiation. More recent studies, however, highlight the massive remodeling of the proteome and metabolome accompanying these events, which is the focus of the review from Marchingo and Cantrell [5]. The authors discuss the general features of protein synthesis and degradation, their dynamic regulation upon $T$ cell activation and differentiation, and the power of high-resolution mass spectrometry technology for proteomic profiling. The authors also describe the crosstalk between protein remodeling and energy metabolism including glycolytic and oxidative metabolism, and the roles of Myc and mTORC1 signaling as central nodes in connecting these processes. Specifically, $\mathrm{T}$ cell activation results in a pronounced increase of Myc expression and mTORC1 activation, which in turn drive the transcriptional and translational upregulation of metabolic enzymes, respectively.

Heintzman, Fisher, and Rathmell discuss how T cell responses and functions are shaped by extrinsic signals from the tissue microenvironment, including macronutrients (e.g., glucose and amino acids), micronutrients (e.g., ions and minerals), and other environmental cues (e.g., temperature and hypoxia) [6]. The authors also describe the regulation and function of $T$ cell immunity in obesity and cancer, with a particular focus on the tumor microenvironment (TME). In the TME, $T$ cell function is impaired by depletion of obligatory metabolites and/or accumulation of immunosuppressive metabolites including kynurenine, adenosine, and lactic acid. Deciphering these processes offers an exciting opportunity to understand tissue immunity and develop innovative strategies for cancer therapy.

Of note, these intrinsic and extrinsic processes in $\mathrm{T}$ cell responses are intimately linked. For example, both protein synthesis and metabolic reprogramming in $\mathrm{T}$ cell activation require active uptake of amino acids, which serve as both building blocks and energy sources [5]. Equally dynamic is the interplay between immunometabolism and innate immunity, as reviewed by Dr. Steven Bensinger [7], Dr. Jenny Ting [8], and colleagues. Analogous to antigen-driven activation of $\mathrm{T}$ cells, macrophages profoundly reprogram cellular cholesterol homeostasis upon engagement of pattern recognition receptors (PRRs) [7]. Lee and Bensinger first provide the overview of cholesterol metabolism, including its synthesis, import, export, and esterification, followed by the emerging concepts for three separate pools of plasma membrane cholesterol encompassing metabolically active, sphingomyelin-sequestered, and essential pools. The authors then discuss how cholesterol metabolism in macrophages is reprogrammed by PRRs and proinflammatory stimuli, and how these changes tune the sensitivity to cholesterol-dependent cytolysins, a family of pore-forming toxins that rely on cholesterol for their effector function. Metabolic coordination of innate immunity therefore serves important roles in infectious and inflammatory diseases.

While immune responses and metabolic programs are known to depend upon signals transduced from surface immune receptors, we are just beginning to understand how intracellular innate immune sensors and receptors intersect with metabolic pathways, as reviewed by Chou, Rampanelli, Li and Ting [8]. The authors discuss how metabolic pathways affect the activities of PRRs including NOD-like receptors (NLRs), AIM2-like receptors, and
STING signaling, and how these intracellular innate immune receptors impact multiple metabolic pathways. For instance, alterations in glycolytic or tricarboxylic acid cycle (TCA) intermediates, mitochondrial activity, or systemic metabolism can perturb the metabolic circuits, leading to the accumulation of specific endogenous metabolites or danger molecules that in turn activate the NLR receptor NLRP3 and induce inflammation. It is important to note that many of the intracellular innate immune receptors can also affect the metabolism and function of $T$ cells and $B$ cells, suggesting their important roles in bridging the innate and adaptive immune systems.

Collectively, these reviews provide new insights into the emerging concept of bidirectional metabolic signaling. Indeed, while it was relatively well understood that immune signaling drives metabolic activation [9], more recent studies reveal that metabolites have signaling roles in both the innate and adaptive immune systems [3]. We also start to unravel the sensing and signaling mechanisms underlying how nutrients license immune cell function and cancer immunity [10-13].

\section{CELLS: METABOLIC CONTROL OF IMMUNE CELL STATE AND FATE}

With the increasing understanding of metabolic signaling, its impacts on immune cell activation state and fate decision are also recognized. Since the initial discoveries of metabolic reprogramming of $T$ cell activation [9, 14] and differentiation [15-17], the past few years have witnessed remarkable advances in the knowledge on metabolic coordination of $T$ cell immunity, as discussed above $[5,6]$. Emerging studies also lead to better understanding of metabolic controls of B cell, DC and macrophage biology, as reviewed by Dr. Mark Boothby [18], Dr. Ping-Chih Ho [19], Dr. David Sancho [20], and colleagues, respectively.

First, Boothby, Brookens, Raybuck, and Cho provide a comprehensive review on immunometabolism of the mature $B$ cell lineage, which culminates in antibody secretion for effective humoral immunity [18]. The authors describe the interplay between nutrients, metabolic programs, and signaling pathways, and their roles in naïve $B$ cell activation, fate choices between cell death, survival, cycling, and terminal differentiation to form plasma cells, and building and fueling the antibody secretion machinery. In particular, the review focuses on nutrient supply (e.g., glucose, glutamine, fatty acids, and oxygen) and the downstream sensors and integration mechanisms that mediate $B$ cell activation and differentiation, and the function of the discrete metabolic programs, including energetic and biosynthetic pathways, reactive oxygen species (ROS), and redox regulation. While nutrient use and bidirectional metabolic signaling are conserved between B cells and T cells, B cells also use many unique programs to support their lineage choices and antibody production.

Second, Moller, Wang, and Ho review metabolic programming in DCs, the key antigen-presenting cells linking innate and adaptive immunity, in immune responses and homeostasis [19]. Several metabolic programs are discussed, including glycolysis, mitochondrial metabolism, lipids, and ER homeostasis, as well as their crosstalk with mTORC1, AMPK, and Hippo kinases. The authors describe these metabolic changes and requirements underlying DC development, activation, maturation, tolerogenic function, and cross-presentation. In particular, they focus on how metabolism controls DC function in the TME and for the interaction with microbiota, as examples of context-specific metabolic rewiring of DC function.

Third, Wculek, Dunphy, Heras-Murillo, Mastrangelo, and Sancho review immunometabolism of tissue macrophages [20]. As tissue-resident immune cells mediating innate immunity and tissue homeostasis, macrophages execute tissue-specific 
specialized functions, and accordingly they adapt their metabolism in response to particular microenvironmental cues, such as nutrients, pathogens, and disease states. The review provides a comprehensive discussion of the diverse metabolic activities and adaptations of macrophages residing in lung, spleen, liver, peritoneum, adipose tissue, brain (microglia), and bone (osteoclasts), and the impacts of macrophage metabolism on tissue repair and regeneration. How dysregulation of macrophage metabolism contributes to chronic pathologies such as overnutrition, atherosclerosis, and cancer, and the therapeutic implications, are also reviewed. In summary, these review articles provide cutting-edge information on how immunometabolism drives immune cell state and fate and contributes to immune function and tissue homeostasis.

\section{SYSTEMS: INTEGRATING INTRACELLULAR NETWORK, INTERCELLULAR CROSSTALK, AND ORGANISMAL IMMUNOMETABOLISM}

One main challenge of studying metabolic pathways is their complexity, which stems from the vast number of metabolites, the diverse chemical structures, and the sophisticated regulation of the enzymes that control them. Similarly, the immune system encompasses heterogeneous cell types, subsets, and activation states, and context-specific interactions with environment and other cells. Therefore, an integrative view is important to understand metabolic control of cellular, tissue, and organismal homeostasis by deciphering the intracellular metabolic network [21], cell-cell interaction between immune and metabolic tissues [22], and the interplay between immune cells and diet [23], as reviewed by Dr. Vijay Kuchroo, Dr. Axel Kallies, Dr. Lydia Lynch, and colleagues, respectively.

Purohit, Wagner, Yosef, and Kuchroo discuss systems-based approaches to study immunometabolism [21]. The authors first discuss experimental approaches to measure cellular metabolism, including metabolic flux analysis, flow cytometry, single-cell RNAsequencing, metabolomics, and CRISPR-based functional genomics screens. The authors then describe computational approaches and mathematical modeling of cellular metabolism, including both kinetic and steady-state approaches, and the applications of these systems-based approaches to study immunometabolism. Specifically, these approaches have been applied to advance the understanding of environment-specific metabolic dependencies of immune cells, identification of novel disease regulators, integration of multiomics for network building, and genome-scale metabolic modeling of patient data. Therefore, systems biology approaches and computational models are powerful tools to interrogate the metabolic network of immune cells and predict disease targets.

While investigations of cellular and organismal metabolism have evolved separately in the space of immunometabolism over the past decade, integrative studies are also emerging. Man, Kallies, and Vasanthakumar review how immune cells regulate adipose tissue homeostasis to control organismal metabolism and thermogenesis [22]. The regulation of systemic metabolism, including organismal glucose homeostasis, insulin sensitivity, and thermogenesis, is dependent upon proper functioning and intricate cell-cell interactions of the adipose tissue, which includes adipocytes, diverse immune cells, and stromal cells. The authors discuss how tissue-resident immune cells preserve insulin sensitivity, how immune cell infiltration into adipose tissue drives insulin resistance, and the adipose tissue niches that mediate immune-stromal cell crosstalk. The authors also discuss the immune and inflammatory basis of obesity and regulation of thermogenesis, and how restoring immune balance in adipose tissue is a promising approach to treat metabolic inflammation and insulin resistance.

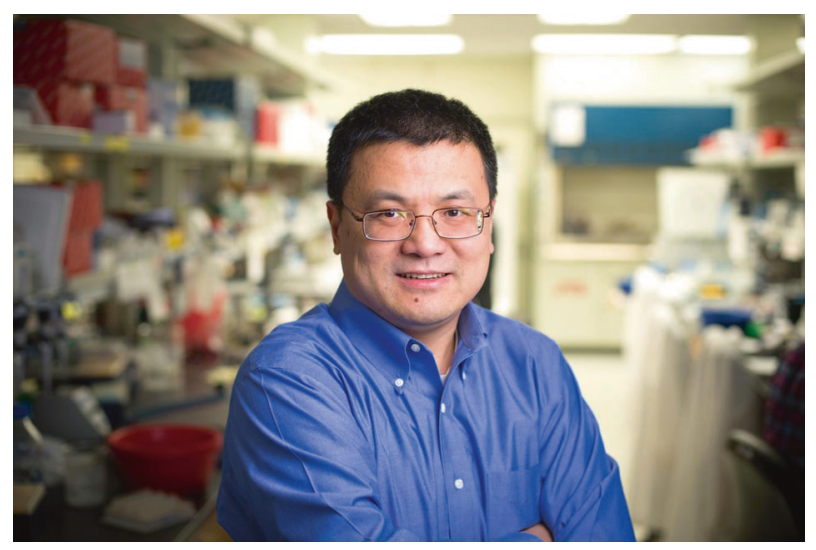

Credit: Dr. Hongbo Chi

Diet plays a crucial role in metabolic homeostasis but can also shape immunity and cancer therapy. Prendeville and Lynch review the roles of lipid metabolism in tumor and immune cells [23]. The authors first provide an overview of lipid uptake, de novo synthesis, and degradation, and their pro-tumorigenic roles in cancer development and metastasis. The authors then discuss the impact of lipids at shaping immune cells in the TME, especially recent studies revealing lipid-mediated inhibition of CD8 T cell and NK cell function but their surprising roles in supporting immunosuppression mediated by regulatory $T$ (Treg) cells, $\gamma \delta$ $T$ cells and tumor-associated macrophages. Given the increased risk of many cancers associated with obesity, understanding the relationship between dietary lipids, tumor cells, and immune cells is important in the context of obesity and cancer therapy. Collectively, these three review articles shed new light on the integration of intracellular network, intercellular interactions, and organismal immunometabolism, and the effects on immunity, metabolism, and cancer.

In conclusion, this series of reviews highlight that the coordination of metabolic programs with nutrient inputs, signal transduction, cell fate, tissue adaptation, and systemic homeostasis is a fundamental event in immunity, physiology, and pathophysiology. Continuous exploration of immunometabolism, especially by interdisciplinary approaches, promises to open new avenues for therapeutic targeting of metabolic pathways to treat human disease.

\section{Hongbo Chi (iD ${ }^{1 \times}$ \\ ${ }^{1}$ Department of Immunology, St. Jude Children's Research Hospital, Memphis, TN, USA. ${ }^{\bowtie e m a i l: ~ h o n g b o . c h i @ s t j u d e . o r g ~}$}

\section{REFERENCES}

1. Chapman NM, Chi H. Metabolic adaptation of lymphocytes in immunity and disease. Immunity. 2022;55:14-30.

2. Lercher A, Baazim H, Bergthaler A. Systemic immunometabolism: challenges and opportunities. Immunity. 2020;53:496-509.

3. Chapman NM, Boothby MR, Chi H. Metabolic coordination of T cell quiescence and activation. Nat Rev Immunol. 2020;20:55-70.

4. Trim WV, Lynch L. Immune and non-immune functions of adipose tissue leukocytes. Nat Rev Immunol. 2021, Epub ahead of print.

5. Marchingo JM, Cantrell DA. Protein synthesis, degradation, and energy meta bolism in T cell immunity. Cell Mol Immunol. 2022;19:303-15.

6. Heintzman DR, Fisher EL, Rathmell JC. Microenvironmental Inffluences on T Cell Immunity in Cancer and Infflammation. Cell Mol Immunol. 2022;19:316-26.

7. Lee MS, Bensinger SJ. Reprogramming cholesterol metabolism in macrophages and its role in host defense against cholesterol-dependent cytolysins. Cell Mol Immunol. 2022;19:327-36.

8. Chou WC, Rampanelli E, Li X, Ting JP. Impact of intracellular innate immune receptors on immunometabolism. Cell Mol Immunol. 2022;19:337-51. 
9. Frauwirth KA, Riley JL, Harris MH, Parry RV, Rathmell JC, Plas DR, et al. The CD28 signaling pathway regulates glucose metabolism. Immunity. 2002;16:769-77.

10. Bian Y, Li W, Kremer DM, Sajjakulnukit P, Li S, Crespo J, et al. Cancer SLC43A2 alters T cell methionine metabolism and histone methylation. Nature. 2020;585:277-82.

11. Reinfeld BI, Madden MZ, Wolf MM, Chytil A, Bader JE, Patterson AR, et al. Cellprogrammed nutrient partitioning in the tumour microenvironment. Nature. 2021;593:282-8.

12. Long L, Wei J, Lim SA, Raynor JL, Shi H, Connelly JP, et al. CRISPR screens unveil signal hubs for nutrient licensing of T cell immunity. Nature. 2021;600:308-13.

13. Huang $H$, Zhou $P$, Wei J, Long $L$, Shi $H$, Dhungana $Y$, et al. In vivo CRISPR screening reveals nutrient signaling processes underpinning $\mathrm{CD} 8(+) \mathrm{T}$ cell fate decisions. Cell. 2021;184:1245-61. e21

14. Wang R, Dillon CP, Shi LZ, Milasta S, Carter R, Finkelstein D, et al. The transcription factor Myc controls metabolic reprogramming upon $T$ lymphocyte activation. Immunity. 2011;35:871-82.

15. Pearce EL, Walsh MC, Cejas PJ, Harms GM, Shen H, Wang LS, et al. Enhancing CD8 T-cell memory by modulating fatty acid metabolism. Nature. 2009;460:103-7.

16. Michalek RD, Gerriets VA, Jacobs SR, Macintyre AN, Maclver NJ, Mason EF, et al. Cutting edge: distinct glycolytic and lipid oxidative metabolic programs are essential for effector and regulatory CD4+ T cell subsets. J Immunol. 2011;186:3299-303.

17. Shi LZ, Wang R, Huang G, Vogel P, Neale G, Green DR, et al. HIF1a-dependent glycolytic pathway orchestrates a metabolic checkpoint for the differentiation of TH17 and Treg cells. J Exp Med. 2011;208:1367-76.

18. Boothby MR, Brookens SK, Raybuck AL, Cho SH. Supplying the trip to antibody production-nutrients, signaling, and the programming of cellular metabolism in the mature B lineage. Cell Mol Immunol. 2022;19:352-69.

19. Moller SH, Wang L, Ho PC. Metabolic programming in dendritic cells tailors immune responses and homeostasis. Cell Mol Immunol. 2022;19:370-83.

20. Wculek SK, Dunphy G, Heras-Murillo I, Mastrangelo A, Sancho D. Metabolism of tissue macrophages in homeostasis and pathology. Cell Mol Immunol. 2022;19:384-408.
21. Purohit V, Wagner A, Yosef N, Kuchroo VK. Systems approaches to study immunometabolism. Cell Mol Immunol. 2022;19:409-19.

22. Man K, Kallies A, Vasanthakumar A. Resident and migratory adipose immune cells control systemic metabolism and thermogenesis. Cell Mol Immunol. 2022;19:420-30.

23. Prendeville H, Lynch L. Diet, lipids, and antitumor immunity. Cell Mol Immunol. 2022;19:431-43.

\section{ACKNOWLEDGEMENTS}

The author acknowledges N. Chapman and H. Shi for scientific discussion and critical reading of the paper. The work in the author's laboratory is supported by ALSAC and grants from NIH and Lupus Research Alliance.

\section{AUTHOR CONTRIBUTIONS}

$\mathrm{HC}$ conceived and wrote the paper.

COMPETING INTERESTS

$\mathrm{HC}$ is a consultant for Kumquat Biosciences.

ADDITIONAL INFORMATION

Correspondence and requests for materials should be addressed to Hongbo Chi.

Reprints and permission information is available at http://www.nature.com/ reprints 